\title{
THE HERBARIUM AND TYPE SPECIMENS OF THOMAS HENRY KEARNEY, JR. FROM I 890-190I *
}

\author{
W. Alan Wentz**
}

\section{Summary}

Specimens of vascular plants designated as types by Thomas $\mathrm{H}$. Kearney, Jr. during 1890 to 1901 have been inaccessible and considered destroyed since the third decade of the twentieth century. These specimens have been found to be extant in the New York Botanical Garden Herbarium, the Ohio State Herbarium, and the United States National Herbarium. The majority of these types are at the Ohio State University which houses T. H. Kearney's original collection of several thousand specimens. The collection is also rich in types which Kearney received from W. W. Ashe, A. W. Chapman, F. V. Coville, A. Gattinger, E. L. Greene, A. A. Heller, F. Lamson-Scribner, C. Mohr, G. V. Nash, C. L. Pollard, A. Ruth, J. K. Small, and other contemporary botanists. The collection at the Ohio State University probably contains additional types which are as yet unverified. This paper catalogues the known type specimens of T. H. Kearney, Jr.

The long and illustrious career of Thomas Henry Kearney, Jr. (1874-1956) extended from the time he published his first scientific paper at the age of 17 (Kearney $189 \mathrm{I}$ ) until his death in 1956 - an incredible 65 years! His life and work have been summarized in Kearney (1958a, 1958b) and McClintock (1958).

Kearney's contributions to taxonomic botany were considerable. Before I 90 I he published names for more than 40 new plant taxa. From I 890 to r90I Kearney collected many thousands of plant specimens in the southeastern United States. In later years his taxonomic investigations encompassed the Malvaceae and the flora of southwestern North America (McClintock 1958). Kearney was well-known to contemporary botanists, many of whom named taxa based on his specimens.

Because of the large number of taxa that are based on Kearney's collections, his specimens are of considerable scientific value. Although many of his specimens (including some types) from the I890-I90 period are known to be at the United States National Herbarium and the New York Botanical Garden, a large number are not at either institution. Unfortunately, that situation led several taxonomists into problems of typification which, more often than not, have proved unsolvable. These taxonomists, Agnes Chase, Carl Epling, Harold Moldenke, and Francis Pennell, to name a few, have variously designated lectotypes, recognized the wrong sheets as types, or ignored names based on Kearney's collections.

While no one in recent years has known the fate of Kearney's collections from the I890-1901 period, the assumption has been that his personal herbarium was deposited at the University of Tennessee where it was

* Contribution from the Botany Department and the Herbarium of the Ohio State University, Columbus, Ohio, 432 Io, U.S.A.

** School of Natural Resources, University of Michigan, Ann Arbor, 48ro4, U.S.A. 
subsequently destroyed in that herbarium's disasterous fire on 18 January 1934 (Andre 197I). In July I97I, I became aware that the Ohio State Herbarium has a large number of Kearney's specimens, several of which have been designated as types by Kearney. With this available information, I proceeded to search the entire vascular plant collection at the Ohio State University to determine the nature and extent of the Kearney collection. It soon became apparent that the Ohio State Herbarium houses the original collection of T. H. Kearney, Jr. from the 1890-190r period.

This paper summarizes the results of herbarium research at the New York Botanical Garden, the Ohio State University, and the U.S. National Herbarium. My evidence shows that the T. $\mathrm{H}$. Kearney specimens at the Ohio State Herbarium represent his original collections, including types, that resulted from his research before the year $190 \mathrm{r}$. Specimens resulting from Kearney's research on the Malvaceae and the southwestern United States flora are not considered in this report.

The evidence that the Ohio State University collection is Kearney's personal herbarium follows:

1. All of the specimen sheets at the Ohio State University are stamped "Herbarium of T. H. Kearney, Jr." while none of the sheets at the New York Botanical Garden or the U.S. National Herbarium are thus stamped.

2. Many of the sheets are accompanied by copious notes in Kearney's handwriting. In addition, original line drawings and/or copies of published papers are placed with some specimens.

3. Several specimens which were designated as new species have new names written on the sheets and taxonomic descriptions prepared and left with them. However, these names were never published.

4. Some of the specimens were marked as types by Kearney. For these he used the following designations (modern equivalents given in parenthesis):

"Type!" (= Holotype)

"Dupl. Type" (= Isotype)

"Co-Type!" (= Syntype)

"Dupl. Co-type" (= Isosyntype).

5. In many cases there are several duplicates of type specimens and other collections.

6. The collection includes duplicate specimens from the Harriman Alaska Expedition (ca. 155 sheets) in which Kearney participated and from Kearney's botanical survey of the Dismal Swamp (Kearney 1901).

7. Many specimens in the collection were sent to Kearney by his contemporaries. In several instances these sheets are also accompanied by short letters or notes.

8. Several of the specimens from the Kearney collection are stamped "From the Herbarium of the University of Tenn." and at least one sheet was annotated at the Ohio State University in the year 1901. I have, therefore, assumed that the Kearney collection was transfered from the University of Tennessee to the Ohio State University during or before the year 1901. (It is probable that his collection contains duplicates of types which were destroyed in the fire at the University of Tennessee.)

Kearney's personal herbarium consists of several thousand sheets and is exceedingly rich in types, not only for taxa he described, but also those which he received as gifts or by exchange from such eminent botanists as William W. Ashe, Alvan W. Chapman, Frederick V. Coville, Augustin Gattinger, Edward L. Greene, A. Arthur Heller, F. Lamson-Scribner, Charles Mohr, George V. Nash, Charles L. Pollard, Albert Ruth, and John $\mathrm{K}$. Small. Many duplicate types for taxa named by these botanists were 
found in Kearney's collection; these have since been verified and enumerated by Stuckey and Wentz (1974). Undoubtedly, additional unmarked types exist in the Kearney herbarium.

ANNOTATED CATALOG OF THE TYPE SPECIMENS OF T. H. KEARNEY, JR. FROM 1890-1901.

This catalog lists the known type specimens of T. H. Kearney, Jr. from the New York Botanical Garden, the Ohio State Herbarium, and the United States National Herbarium. I have verified the types at New York and Ohio. The data from the U.S. National Herbarium were supplied by R. L. Stuckey of the Ohio State University. Those types which remain unlocated are possibly at the U.S. National Herbarium. Additional duplicates and paratypes are probably also at the U.S. National Herbarium and possibly at other institutions.

The catalog is arranged alphabetically by family and alphabetically by species within each family. For each taxon named by Kearney a complete citation followed by the specimen data and its location is given. Additional notes on types and new combinations have occasionally been included in order to clarify problems. Unpublished names have been indicated only by asterisk. In most cases it has been impossible to relate these unpublished names to the currently applied name. Capitalization of specific epithets and abbreviations of author's names are given as published by Kearney. Herbarium acronyms follow Lanjouw and Stafleu (1964).

ANACARDIACEAE

Rhus Blodgettii Kearney, Bull. Torrey Bot. Club 2I: 486-7. I 894.

HOLOTYPE: FLORIDA: Pine Key, Key West, [date unknown] Blodgett s.n., NY; det. as Toxicodendron radicans (L.) Kuntze by F. A. Barkley, 1937. Specimen from the Torrey Herbarium. Photograph of specimen available from Photo. Lab. Info. Services, Michigan State University, East Lansing, Negative number 631978-I. ARISTOLOCHIACEAE

Aristolochia Nashii Kearney, Bull. Torrey Bot. Club 21: 485-6. I 894.

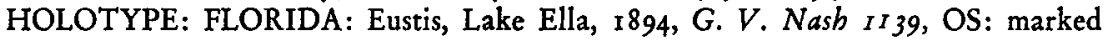
"type" by Kearney.

COMPOSITAE

Pluchea foetida (L.)DC. var. imbricata Kearney, Bull. Torrey Bor. Club 21:483-4. I 894 .

HOLOTYPE: FLORIDA: Eustis, July ı 894, G. V. Nash 1434, OS; marked "type" by Kearney; ISOTYPES: NY -2 sheets, OS -2 sheets.

Solidago *****

NORTH CAROLINA, 3 sheets at OS marked as "new species" by Kearney, but never published.

Xanthium speciosum Kearney, Bull. Torrey Bot. Club 24:574-5. 1897.

HOLOTYPE: TENNESSEE: Cocke county, French Broad River, between Point Rock and Del Rio, 16 Sep $1897, T$. H. Kearney, Jr. 785, OS; ISOTYPES: MO, NDG, NY, US (see: C. F. Millspaugh and E. E. Sherff. 1919. Revision of the North American Species of Xanthium. Field Mus. Nat. Hist. Bot. Ser. 4(2): 9-52).

Xanthium

MISSISSIPPI: Clay county, 21 Sep r89I, A. B. Seymour, s.n., 4 sheets at OS marked "new species"; one marked "type" but never published.

Xantbium $* * * * *$

VIRGINIA: Princess Anne county, Cape Henry, 6 Oct 1898, T. H. Kearney, Jr. 2124, OS, marked "an undescribed species" by Kearney but never published.

ERICACEAE

Vaccinium melanocarpum (C. Mohr) Kearney, Bull. Torrey Bot. Club 24: 570. 1897. New combination based on $V$. stamineum melanocarpum $C$. Mohr, Bull. Torrey Bot. Club. 24: 25. 1897. The name is often erroneously cited as V. melanocarpum Kearney. 
Agrostis intermedia Scribn. in Kearney, Bull. Torrey Bot. Club 20:476. I893. SYNTYPE(S): KENTUCKY: Harlan and Bell counties, 1893, T. H. Kearney, Jr. 39, OS, US-?

Andropogon scoparius Michx. var. villosissimus Kearney in Scribn. and Ball., U.S.D.A.

Div. Agrost. 24: 4I. r9or.

HOLOTYPE: MISSISSIPPI: Waynesboro, 2 Oct 1896, T. H. Kearney, Jr. 136, US; ISOTYPE: OS.

Aristida purpurascens Poir. in Lam. var. glaucissima Kearney in Scribn. and Ball., U.S.D.A. Div. Agrost. 24: 45 . 1900.

HOLOTYPE: MISSISSIPPI: Biloxi, 7 Oct 1896, T. H. Kearney, Jr. 32t, US; ISOTYPE: NY.

Calamagrostis alaskana Kearney, U.S.D.A. Div. Agrost. I I : 32. 1898.

HOLOTYPE: ALASKA: Yukon River, Aug I893, F. Funston I57, US; ISOTYPE: OS, marked "dupl. Type!" by Kearney.

Calamagrostis aleutica Trin. in Bong. var. patens Kearney, U.S.D.A. Div. Agrost. I I: 20. 1898 .

HOLOTYPE: CALIFORNIA: Mendocino, [date unknown] H. N. Bolander s.n., $\mathrm{GH}$.

Calamagrostis breviseta (A. Gray) Scribn. var. debilis Kearney, U.S.D.A. Div. Agrost. II: 25.1898 .

HOLOTYPE: CANADA, NEWFOUNDLAND: Exploits River near mouth of Badger Brook, I 3 Aug I 894, B. L. Robinson \& H. Scbrenk 205, US; PARATYPE: CANADA, NEWFOUNDLAND: Chimney Cove, $20 \mathrm{Jul}$ i $895, A$. Waghorn 8 , OS. Calamagrostis breviseta (A. Gray) Scribn. var. lacustris Kearney, U.S.D.A. Div. Agrost. II : $25-6$. I 898 .

HOLOTYPE: MINNESOTA: Fond du Lac, $23 \mathrm{Jul}$ r889, F. F. Wood s.n., US; PARATYPES: MINNESOTA: Gunflint Lake, i89r, F. F. Wood s.n., NY; MICHIGAN: Isle Royale, r865, Porter s.n., NY; NEW YORK: Racket Lake, I865, Leggett s.n., NY.

Calamagrostis californica Kearney, U.S.D.A. Div. Agrost. I I: 37-8. I898. HOLOTYPE: CALIFORNIA: Sierra Nevada Mtns., I875. J. G. Lemmon 444, US. Calamagrostis canadensis (Michx.) Beauv. var. campestris Kearney, U.S.D.A. Div. Agrost. I I : 3 I. I 898.

HOLOTYPE: CANADA: Assiniboia, Louis Plain, Jul r883, J. M. Macoun 56, US.

Calamagrostis fasciculata Kearney, U.S.D.A. Div. Agrost. II: 23-4. 1898 . HOLOTYPE: CALIFORNIA: Mendocino, Aug 1882, C. G. Pringle s.n., US.

Calamagrostis foliosa Kearney, U.S.D.A. Div. Agrost. I I : 17-8. 1898. HOLOTYPE: CALIFORNIA: Humboldt county, Mattole District, [date unknown] $H$. N. Bolander 6470, US; ISOTYPES: NY, OS-marked "Dupl. Type!" by Kearney.

Calamagrostis hyperborea Lange var. elongata Kearney, U.S.D.A. Div. Agrost. II: 40-1. 1898.

HOLOTYPE: NEBRASKA: Plummer county, Plummer Ford on Dismal River, 1893, P. A. Rydberg 1494 , US; ISOTYPE: NY; PARATYPES: WASHINGTON: Douglas county, Jun 1893 , Sandberg \& Leiberg, 325 , OS; MONTANA: Sheep Creek, 8 Aug 1896, $P$. A. Rydberg 3309, OS.

Calamagrostis byperborea Lange var. stenodes Kearney, U.S.D.A. Div. Agrost. 11: 39. 1898 .

HOLOTYPE: COLORADO: Saguache county, Marshall Pass, i $896, F$. E. Clements 206, US; PARATYPE: MONTANA: Lima, S Aug. I895, $P$. A. Rydberg 2318 , OS.

Calamagrostis inexpansa A. Gray var. barbulata Kearney, U.S.D.A. Div. Agrost. I1: 37. 1898 .

HOLOTYPE: WASHINGTON: Mason county, 26 July i 890, C. V. Piper 947, US. Calamagrostis inexpansa A. Gray var. cuprea Kearney, U.S.D.A. Div. Agrost. II: 37. 1898 .

HOLOTYPE: WASHINGTON: Falcon Valley, 9, 18 Jul i 866, W. N. Suksdorf 910, US; ISOTYPE: NY.

Calamagrostis labradorica Kearney, U.S.D.A. Div. Agrost. II: 38-9. I898. 
HOLOTYPE: CANADA, LABRADOR: Bonne Esperance, I882, J. A. Allen I8, US; ISOTYPE: NY.

Calamagrostis laxiflora Kearney, U.S.D.A. Div. Agrost. II: 34 . 1898.

HOLOTYPE: WYOMING: Yellowstone National Park, East Fork of the Yellowstone River, Aug. 1885, F. Tweedy 582, US.

Calamagrostis lemmoni Kearney, U.S.D.A. Div. Agrost. II: 16-7. I898.

HOLOTYPE: CALIFORNIA: i87s, J. G. Lemmon s.n., US; ISOTYPES: NY, OS- marked "dupl. Type!" by Kearney.

Calamagrostis micrantha Kearney, U.S.D.A. Div. Agrost. II: 36 . 1898.

HOLOTYPE: CANADA, SASKATCHEWAN: n of Prin[c]e Albert, Jul 1896, J. Macoun ${ }_{13 I I I}$ G.S.C., US.

Calamagrostis neglecta (Ehrh.) Gaertn. var. candidula Kearney, U.S.D.A. Div. Agrost. II: $35-6.1898$.

HOLOTYPE: CANADA: Assiniboia, Cypress Hills, 20 June 1894, J. Macoun s.n., in Herb. Geol. Survey Dep. of Canada 7483 [not seen].

Calamagrostis neglecta (Ehrh.) Gaertn. var. wrightii Kearney, U.S.D.A. Div. Agrost. II: 36. I898.

HOLOTYPE: BEARING STRAITS: Arakamchetchene Island, $1853^{-6}$, C. Wright s.n., US.

Calamagrostis nemoralis Kearney, U.S.D.A. Div. Agrost. II: 26-7. 1898.

HOLOTYPE: NEW YORK, Thacher's Pinnacle, Danby, near Ithaca, I Aug. I884, W. Dudley s.n., US.

Calamagrostis scopulorum Jones var. lucidula Kearney, U.S.D.A. Div. Agrost. I I: 33. I 898 .

SYNTYPE(S): UTAH: Altah, Wahsatch Mtns., i 879, M. E. Jones 1145, NY, US-?

Calamagrostis subflexuosa Kearney, U.S.D.A. Div. Agrost. II: 22. I898.

HOLOTYPE: CALIFORNIA: Oakland, [date unknown] H. N. Bolander 2274, US; ISOTYPES: NY-2 sheets, OS- marked "dupl. Type!" by Kearney.

Calamagrostis suksdorfii (Scribn.) Scribn. in Vasey var. luxurians Kearney, U.S.D.A.

Div. Agrost. I I : 24-5. I898.

HOLOTYPE: IDAHO: Lake Coeur d'Alene, Farmington Landing, I892, Sandberg, Heller, and McDougal 630, US; ISOTYPES: NY, OS; PARATYPE: IDAHO: Palouse country and Lake Coeur d'Alene, Jun-Jul I892, G. B. Aiton $4 I$, OS.

Calamagrostis vilfaeformis Kearney, U.S.D.A. Div. Agrost. II: 20 . I898.

HOLOTYPE: CALIFORNIA: San Diego county, Julian, I889, C. R. Orcutt s.n., US; ISOTYPE: OS; PARATYPES: CALIFORNIA: San Diego county, Potrero Valley, ro Jun $\mathrm{I} 890, C$. $R$. Orcutt s.n., OS- specimen consists of inflorescence fragments mounted with isotype; San Diego county, Januel Valley, to Jul I890, C. $R$. Orcutt s.n., NY.

Panicum capillare L. var. geniculatum Scribn. in Kearney, Bull. Torrey Bot. Club 20: 477. 1893 .

SYNTYPES: KENTUCKY: Bell county, Cumberland River, Sep 1893, T. $H$. Kearney, Jr. 335, OS, US -OS specimen det. as P. gattengeri Nash by C. G. Weishaupt, 1969.

Panicum commutatum (Muhl.) Schult. var. latifolium Scribn. in Kearney, Bull. Torrey Bot. Club 20: 476. 1893 .

Scribner apparently did not designate a type for this taxon. However, Hitchcock and Chase (Manual of Grasses, p. 914, 1950) stated that Kearney's specimen number 299 (Kentucky, Pine Mtn., Harlan and Bell counties, 1893) is the type. I have not located this specimen but it is probably at US.

Triodia drummondii Scribn. \& Kearney, U.S.D.A. Div. Agrost. 4: 37.1897.

SYNTYPE(S): MISSISSIPPI: Biloxi, 7 Oct x 896, T. H. Kearney, Jr. 324, OS, US-? -OS specimen det. as Tridens carolinianus (Steud.) Henr. by C. G. Weishaupt.

HAMAMELIDACEAE

Fotbergilla parviflora Kearney in Small, FI. SE U.S. 509. I 903.

According to Small (FL. SE U.S., 1903), type collected by T. H. Kearney, Jr. on 4 June 1893 at Jesup, Georgia and deposited in the Herbarium of Columbia University (now NY). Specimen not located at NY or OS. 
Scutellaria integrifolia L. var. multiglandulosa Kearney, Bull. Torrey Bot. Club 21: 482. 1894 .

SYNTYPES: FLORIDA: Lake county, Eustis, Jul I894, G. V. Nash II26, NY, det. as Isotype of $S$. multiglandulosa Small by C. Epling, I 938 (S. multiglandulosa (Kearney) Small is actually a new combination based on Kearney's variety); St. Mark's, May 1843, Rugel s.n., NY, det. as S. integrifolia L. subsp. bispida (Benth.) Epl. by C. Epling, 1938.

Scutellaria venosa Kearney, Bull. Torrey Bot. Club 24: 571-2. I897.

HOLOTYPE: TENNESSEE: Summit of Bluff Mtn., near Lemon's Gap, 28 Aug I 897, T. H. Kearney, Jr. 873; OS; ISOTYPE: US, det. as S. ovata Hill. subsp. venosa (Kearney) Epl. by C. Epling, 1938.

Tencrium Nashii Kearney, Bull. Torrey Bot. Club $21: 484$. 1894 .

HOLOTYPE: FLORIDA: Eustis, 1894, G. V. Nash 1516, OS- marked "Type" by Kearney; PARATYPES: FLORIDA: Eustis, 1894, G. V. Nash 1496, NY; Duval county, [date unknown] $A$. $H$. Curtiss 1975 , NY; 1836, Chapman 96, NY, all 3 det. as $T$. canadense L. var. angustatum Gray by E. McClintock, 1945.

Trichostema suffrutescens Kearney, Bull. Torrey Bot. Club 21: 483 . I894.

HOLOTYPE: FLORIDA: Eustis, I894, G. V. Nash 625, OS- marked "Type" by Kearney; ISOTYPE: NY.

LEGUMINOSAE

Stylosanthes riparia Kearney, Bull. Torrey Bot. Club 24: 565-8. 1897 .

SYNTYPES: TENNESSEE: Cocke county, French Broad River between Paint Rock and Del Rio, I Sep 1897, T. H. Kearney, Jr. 674, NY, OS- 2 sheets, US- fragment only; Cocke county, French Broad River near Wolf Creek, 2 Aug 1894, $T$. $H$. Kearney, Jr, s.n., OS.

PRIMULACEAE

Dodecatheon pauciflorum Greene var, *****

ALASKA: Point Gustavus, Glacier Bay, ro to 12 Jun $1899, F . V$. Coville $E T$. $H$. Kearney, Jr. 747, (Collected on Harriman Alaska Expedition). Specimen at OS marked "new species" but never published.

Steironema intermedium Kearney, Bull. Torrey Bot. Club 21: 264. 1894. SYNTYPES: ALABAMA: Talladega county, Alpine Mountain, 25 Sep 1892, C. Mobr s.n., NY; Talladega county, Alpine Mountain, Jul r893, C. Mobr s.n., OS; KENTUCKY: Harlan county, Pine Mountain, Aug I893, T. H. Kearney, Jr, x2I, NY, OS- 2 sheets, US; TENNESSEE: Knox county, Jul I89r, T. H. Kearney, Jr. s.n., OS; Knox county, 9 Jul 1894, T. H. Kearney, Jr. s.n., NY- 2 sheets, OS- 2 sheets.

Steironema tonsum Bicknell var. simplex Kearney, Bull. Torrey Bot. Club 24: 571. I897. HOLOTYPE: TENNESSEE: Cocke county, Wolf Creek Station, 6 Sep 1897 , T. H. Kearney, Jr. 83r, OS; ISOTYPE: NY, det. as Lysimachia tonsa (Wood)Knuth by James D. Ray, Jr., I95I.

RANUNCULACEAE

Cimicifuga rubifolia Kearney, Bull. Torrey Bot. Club 24: s61-2. I 897 .

SYNTYPES: TENNESSEE: Knoxville, 8 Sep r890, T. H. Kearney, Jr. s.n., NY, OS, NY specimen det. as lectotype by G. W. Ramsey, r965; Tennessee River near Knoxville, Oct $1897, A$. Ruth s.n., NY, OS- 2 sheets; Knoxville, 6 Sep 189 r, $T$. $H$. Kearney, Jr. s.n., OS.

ROSACEAE

Agrimonia mollis (T. \& G.) Britton var. Bicknellii Kearney, Bull. Torrey Bot. Club 24: 565 . 1897 .

HOLOTYPE: TENNESSEE: Cocke county, Wolf Creek, 2 Sep 1897, T. H. Kear$n e y, J r .69 r$, OS; ISOTYPES: NY, US.

SOLANACEAE

Physalis arenicola Kearney, Bull. Torrey Bot. Club $2 \mathrm{I}: 485$. 1894.

HOLOTYPE: FLORIDA: Eustis, 1894, G. V. Nash 1170, OS- marked "Type" by Kearney; ISOTYPES: NY -2 sheets. 


\section{VERBENACEAE}

Lippia maritima Kearney ex Moldenke, Résumé of Verbenaceae, Avicenniaceae, Stilbaceae, Symphoremaceae, and Eriocaulaceae of the World. p. 314. 1959. pro syn. = Phyla lanceolata (Michx.) Greene.

VIRGINIA: Princess Anne county, Virginia Beach, 3 Aug 1898, T. H. Kearney, Jr. 2033, OS. Kearney did not publish this name, but Moldenke apparently recognized the name from herbarium sheets (at US?).

\section{Acknowledgements}

I am indebted to Ronald L. Stuckey of the Ohio State University, for assistance during this project. Dennis Anderson and Robert R. Haynes, both of the Ohio State University, read the manuscript and suggested helpful changes. Financial support for travel to the New York Botanical Garden was supplied by the Ohio State University College of Biological Sciences.

\section{References}

ANDRE, J. E. 1971 - The history of systematic botany in Tennessee: I770-1942. M. Sc. Thesis. Univ. Tenn. I 8 p.

Kearney, T. H., Jr. 1891 - Cleistogamy in Polygonum acre. Bot. Gaz. I6: 314.

KEARNEY, T. H., Jr. I90x - Report on a botanical survey of the Dismal Swamp region. Contrib. U.S. Natl. Herbarium s: $321-550$.

Kearney, T. H., Jr. 1958a - Autobiographical notes. Leaf. West. Bot. 8(1 2): 273-275.

KeARNEY, T. H., Jr. I958b - Botanists I have known. Leaf. West. Bot. 8(12): $275-280$.

Lanjouw, J. and F. A. Stafleu 1964 - Index Herbariorum. Part I. The herbaria of the world. 5 th. ed. Regnum Vegetabile $3 x$ : $1-25$ I.

McClintock, E. 1958 - Bibliography of the scientific writings of Thomas H. Kearney. Leaf. West. Bot. 8(12): 280-287.

Stuckey, Ronald L. and W. Alan WeNTz I974 - Vascular plant type specimens in the Ohio State University herbarium. Ohio J. Sci. 74: (in press). 